\title{
Dopaminerge und serotonerge Störungen
}

D opaminerge und serotonerge Störungen lautet der Schwerpunkt dieses Heftes. „Dopaminerg“ und „serotonerg“" sind dabei in Anführungszeichen zu setzen, handelt es sich doch bei einer derartigen $\mathrm{Zu}$ ordnung um eine starke Vereinfachung. Gemeint sind Störungen, bei denen das dopaminerge bzw. serotonerge System pathophysiologisch eine maßgebliche Rolle spielt oder, anders formuliert, Störungen, die hinsichtlich der genannten Neurotransmittersysteme pharmakotherapeutische Ansatzpunkte bieten.

Die so charakterisierten neurobiologischen Hypothesen zur Genese bestimmter Störungen beruhen ursprünglich auf empirischen Erkenntnissen der Pharmakotherapie, d.h. Feststellungen über die Wirksamkeit von Psychopharmaka mit bestimmten Wirkmechanismen bei bestimmten Störungen (typisches Beispiel: die aus der Wirksamkeit klassischer Neuroleptika abgeleitete Dopamin-Hypothese der Schizophrenie). Erst später erlaubten neurobiologische Forschungsansätze (vor allem funktionelle bildgebende Verfahren wie die funktionelle Kernspintomographie, die Positronenemissionstomographie und andere) differenziertere Einblicke in die komplexen Zusammenhänge zwischen neurobiologischen Funktionssystemen und der Interaktion verschiedener Neurotransmittersysteme einerseits sowie der Entwicklung von Krankheitsprozessen bis zur Manifestation einer psychiatrischen Störung andererseits.

Von Bedeutung in diesem Zusammenhang ist des weiteren die Frage: Orientiert man sich bei der Konzeption einer pharmakotherapeutischen Vorgehensweise an einer Diagnose im Sinne einer nosologischen Entität oder an einem „Zielsyndrom“ (der Begriff „Störung“ bzw. "disorder" in den neueren Klassifikationssystemen nimmt hier in gewisser Weise eine Zwischenstellung ein) oder eher an einem „Ziel“- oder „Leitsymptom“? Der Trend in der klinischen Psychiatrie in den letzten Jahren geht in letztgenannte Richtung, genauer formuliert: Man orientiert sich an mutmaßlichen pathophysiologischen Mechanismen, die einen (evidenzbasiert) effizienten pharmakotherapeutischen Ansatzpunkt bieten, womit der Kreis zum Ausgangspunkt dieser Betrachtung wieder geschlossen wäre.

Als Beispiel für eine „dopaminerge Störung“ wird in diesem Heft der aktuelle Wissensstand über Tic-Störungen dargelegt. Ein zentraler pathophysiologischer
Mechanismus besteht hierbei in einer erhöhten Exzitabilität des sensomotorischen Systems bzw. in einer unzureichenden motorischen Inhibition auf subkortikaler Ebene. Dopamin-2-Rezeptor-Blocker stellen hier ein wirksames pharmakotherapeutisches Behandlungsprinzip dar, um diesem Ungleichgewicht entgegen zu wirken. Bei Zwangsstörungen sind, bei entsprechender genetischer bzw. neurobiologischer Disposition, inadäquate Entwicklungs-, Lern- und Anpassungsprozesse (pathologische gebahnte Erregungsmuster) von wesentlicher Bedeutung. Nach heutigem Wissensstand ist nicht von einem Serotoninmangel als Ursache von Zwangsstörungen auszugehen, vielmehr handelt es sich eher um eine komplexe zentralnervöse Netzwerkstörung, wobei dem Serotonin wohl eine modulatorische Funktion zukommt. Einen wirksamen pharmakotherapeutischen Ansatzpunkt bietet das serotonerge System hierbei für Substanzen, welche die Serotonin-Wiederaufnahme im synaptischen Spalt hemmen. Dies führt in der Regel zu einer Symtomreduktion und erleichtert in Verbindung mit verhaltenstherapeutischen Maßnahmen auf neurobiologischer Ebene den Aufbau neuer, adäquater Verhaltensmuster. Eine besondere therapeutische Herausforderung stellen komorbide Störungen dar. Neurobiologische und pharmakotherapeutische Überlegungen bezüglich klinisch relevanter komorbider Störungen (unter besonderer Berücksichtigung des Dopamin- und des SerotoninSystems) werden in einem weiteren Beitrag angestellt.

Je mehr unser bislang noch recht begrenztes Wissen um die komplexen Zusammenhänge und Interaktionen auf neurobiologischer und neuropsychologischer Ebene voranschreitet, um so differenziertere und effektivere therapeutische Strategien werden sich entwickeln lassen.

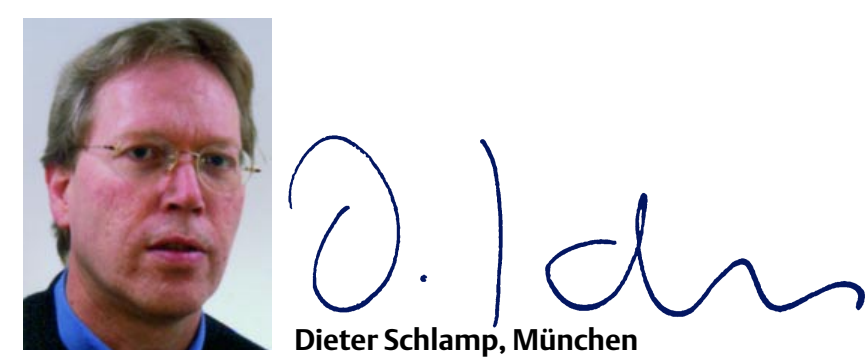

